\section{Personalized medicine: paradigm shift or revolution}

To the Editor: I am writing this letter in response to Marcon et al., "Representing a 'revolution': how the popular press has portrayed personalized medicine."

The authors maintain that the terms "personalized medicine (PM)" or "precision medicine" have different definitions in popular press articles, or "mass media," and are partly responsible for its positive and optimistic portrayal. However, a distinction between "definition" and "meaning" must be made in this context. While there do exist differences in the definition of personalized and precision medicine, its meaning for the various stakeholders in health care is more relevant. The meaning of PM to providers (in both major medical centers and community hospitals), payers, patients, the biotech/medical device/pharma industries, and the general audience (that this paper analyzes) are also varied, but with differing results. For providers, PM offers opportunities for administering effective, efficient, and cost-saving care to patients. Payers have been less than cooperative in paying for PM services (undermining possibly the general premise of the article that PM portrays a optimistic or rosy picture for the public), because payers mostly rely on evidence-based studies that would convince them to pay for genetic testing and lifesaving targeted therapies. Industry has made changes as well to its business models to adapt to the gains made in PM for health care.

Furthermore, the article characterizes PM as a "revolution" and "hype." A more accurate characterization may be "paradigm shift." The phenomenon of the paradigm shift was originated by historian of science Thomas Kuhn, who argued that scientific revolutions occur through paradigm shifts. That is, the scientific community at first resists the shift, but as more of its community members embrace the shift due to the weight of evidence corroborated with experiments, both theoretical and empirical, gradually the shift becomes adopted. These sources should form the basis of whether personalized medicine is portrayed optimistically or positively with its concerns addressed in the literature. This literature should come in the form of well-referenced and well-cited sources, and the additional weight of evidence for this would come from precision-based clinical trials, such as the National Cancer Institute Molecular Analysis for Therapy Choice trial (NCI-MATCH) and I-SPY.

It is accurate for the authors to claim that personalized medicine impact's on health care lies mainly in cancer, diabetes, cardiovascular disease, and cystic fibrosis. However, we have not yet reached the apex of health care for these existing trends to continue and have not yet been achieved for precision or personalized medicine, and would provide additional medical and economic benefits to patients. One example is in the area of genome-wide associated studies; others include helping to solve diagnostic odysseys, developing more targeted drugs, and implementing the Institute of Medicine's Knowledge Infrastructure Initiative. Finally, the article does not mention some of the actual concerns of PM, such as issues surrounding data sharing with researchers who have been held liable for misuse of data from vulnerable communities.

\section{DISCLOSURE}

P.H. declares no conflicts of interest.

\section{Priya Hays, $P h D^{1}$}

${ }^{1}$ Hays Documentation Specialists LLC, San Mateo, CA, USA.

Correspondence: Priya Hays (priya@haysdocumentationspecialists.com)

\section{REFERENCE}

1. Marcon AR, Bieber M, Caulfield T. Representing a "revolution": how the popular press has portrayed personalized medicine. Genet Med. 2018;20:950-956.

Advance online publication 23 November 2018. doi:10.1038/s41436-018-0379-8 